Pacific Journal of Mathematics

MULTIPLICATIONS ON HOMOGENEOUS SPACES, 


\title{
MULTIPLICATIONS ON HOMOGENEOUS SPACES, NONASSOCIATIVE ALGEBRAS AND CONNECTIONS
}

\author{
A. A. SAgle AND J. R. Schumi
}

In this paper we show how nonassociative algebras over the real numbers arise from multiplications on certain homogeneous spaces; that is, an analytic function $\mu: M \times M \rightarrow M$. Then these algebras are used to obtain an invariant connection $\nabla$ on the homogeneous space and we give some applications of nonassociative algebras to these topics. Conversely every finite dimensional nonassociative algebra over the real numbers arises from an invariant connection and a local multiplication on a homogeneous space. Thus, analogous to the theory of Lie groups and Lie algebras, much of the basic theory of nonassociative algebras can be formulated in terms of multiplications and connections and conversely.

1. Introduction. Let $G$ be a connected Lie group with Lie algebra $g$ and let $H$ be a closed subgroup with Lie algebra $h$. Then the pair $(G, H)$ or $(g, h)$ is called a reductive pair if there exists a subspace $m$ of $g$ so that $g=m+h$ (subspace direct sum) and $(\operatorname{Ad} H)(m) \subset m$. The corresponding homogeneous space $G / H$ is called a reductive homogeneous space which is an analytic manifold. An analytic function

$$
\mu: G / H \times G / H \longrightarrow G / H
$$

such that $\mu(\bar{e}, \bar{e})=\bar{e}=e H$ is called a multiplication on $G / H$; for example, Lie groups, Moufang loops and certain $H$-spaces are reductive homogeneous spaces with a multiplication.

The nonassociative algebras arise from studying the local behavior of a multiplication $\mu$ on $G / H$ which we now consider. Thus let $\pi$ : $G \rightarrow G / H$ be the natural projection and let $g=m+h$ be a fixed (reductive) decomposition. From [1, p. 113] we know that for the $\operatorname{map} \psi=\exp \mid m$ there exists a neighborhood $U$ of 0 in $m$ which is mapped homeomorphically into $G$ under $\psi$ and such that $\pi$ maps $\psi(U)$ homeomorphically onto a neighborhood $N^{*}$ of $\bar{e}$ in $G / H$. Thus by the analyticity of $\mu$ and $\pi \circ \psi$ there exists a neighborhood $D$ of 0 in $m$ contained in $U$ so that for all $X, Y \in D$

$$
\mu(\pi \exp X, \pi \exp Y)=\pi \exp F(X, Y)
$$

is in $N^{*}$ where $F: D \times D \rightarrow U$ is a function which is analytic at $\theta=$ $(0,0) \in m \times m$. Thus $\mu$ is determined locally by $F$ which has the Taylor's series expansion [5] $F(X, Y)=F(\theta)+F^{1}(\theta)(X, Y)+1 / 2 F^{2}(\theta)(X, Y)^{2}+$ 
... for $X, Y \in D$. We will show that $F(\theta)=0$ and the function

$$
\alpha(X, Y)=F^{2}(\theta)[(X, 0),(0, Y)]
$$

is bilinear. Therefore the multiplication $\mu$ on $G / H$ determines a nonassociative algebra with linear space $m$ and composition $\alpha: m \times$ $m \rightarrow m$ and we denote this algebra by $(m, \alpha)$.

Next we require that for all $u \in H$ the mappings

$$
\tau(u): G / H \longrightarrow G / H: y H \longrightarrow u y H
$$

are automorphisms of the multiplication $\mu$ on $G / H$, and call the pair $(G / H, \mu)$ with $\tau(H) \subset \operatorname{Aut}(G / H, \mu)$ a multiplicative system. We show that $\tau(H) \subset \operatorname{Aut}(G / H, \mu)$ implies $\operatorname{Ad} H$ is in the automorphism group of the algebra $(m, \alpha)$ and this allows us to use the result [6] which gives a bijective correspondence between $G$-invariant connections $\nabla$ on $G / H$ and nonassociative algebras $(m, \alpha)$ with $\operatorname{Ad} H \subset \operatorname{Aut}(m, \alpha)$. Thus a multiplicative system $(G / H, \mu)$ induces a $G$-invariant connection via the algebra $(m, \alpha)$. Conversely, we show that every such algebra comes from a local multiplicative system. In particular, any finite dimensional nonassociative algebra $A$ over $R$ can be regarded as an algebra $(g, \alpha)$ for a Lie algebra $g$ of suitable dimension and consequently $A$ arises from a local multiplicative system defined on $G$ and also from a $G$-invariant connection defined on $G$.

The above multiplicative systems $(G / H, \mu)$ are too general and not particularly related to the action of $G$ on $G / H$. We will now describe an "invariance" restriction for the multiplicative system $(G / H$, $\mu)$. The multiplication $\mu$ defines a function $\iota(\mu, X): G / H \rightarrow T(G / H)$ from $G / H$ to the tangent bundle of $G / H$ as follows; (see [9] for the case of Lie groups). Let $T(G / H, \bar{a})$ denote the tangent space at $\bar{a} \in$ $G / H$; thus $T(G / H, \bar{e})=m$. Let $T$ denote the differential of a function, then for each $X \in m$ we set

$$
\iota(\mu, X)(\bar{a})=[T \mu(\bar{a}, \bar{e})](0, X) \text { for }(0, X) \in T(G / H, \bar{a}) \times m .
$$

That is, $\ell(\mu, X)(\bar{\alpha})$ is the differential of $\mu$ evaluated at $(\bar{a}, \bar{e})$ on $(0$, $X)$. The function $\ell(\mu, X)$ is a vector field if and only if $(G / H, \mu)$ has $\bar{e}$ as a right identity element; also the vector field is analytic and depends linearly on $X$. For $\Gamma$ a subset of $\tau(G)$ containing $\tau(e)$, whose precise definition will be given in $\S 4$, we say that $\mu$ is $\Gamma$-invariant if for all $X \in m, \ell(\mu, X)$ is a vector field invariant under all the maps $\tau(a): G / H \rightarrow G / H: \bar{x} \rightarrow a \bar{x}$ for all $\tau(a) \in \Gamma$. That is, for all $\tau(a) \in \Gamma$, and all $X \in m, \ell(\mu, X)$ satisfies

$$
T \tau(a)(\bar{e}) \ell(\mu, X)(\bar{e})=\ell(\mu, X)(\tau(a) \bar{e}) .
$$

In particular if $(G, \mu)$ is a Lie group, then $\Gamma$ can be taken to be 
$L(G)=\{L(a): a \in G\}$ and $\ell(\mu, X)$ is the usual left invariant vector field generated by $X$, so our results are consistent with Lie theory. In the case of a $\Gamma$ invariant multiplication $\mu$, we obtain the connection induced by $\mu$ is given by the algebra $(m, \alpha)$ with $\alpha(X, Y)=F^{2}(\theta)[(X$, $0),(0, Y)]=1 / 2[X Q Y]$ where $Q$ is the endomorphism of $m$ given by $Q: m \rightarrow m: Y \rightarrow F^{1}(\theta)(0, Y)$. Although we have used a global multiplication in the above discussion, most of the results concern the algebra $(m, \alpha)$, thus it suffices to consider local multiplications on $G / H$. However the globalization of these local results present many topological problems. For example, every sphere is a reductive homogeneous space $G / H$ and consequently has in a suitable neighborhood of $\bar{e}=e H$ a local multiplication with $\bar{e}$ as an identity element. Thus any sphere is a local $H$-space but only $S^{1}, S^{3}$, and $S^{7}$ are global $H$-spaces.

2. Multiplications. Using the notations of $\S 1$ we have for $X$, $Y$ in a suitable neighborhood $D$ of 0 in $m$ that

$$
\mu(\pi \exp X, \pi \exp Y)=\pi \exp F(X, Y)
$$

where $F: D \times D \rightarrow U$ is analytic at $\theta=(0,0) \in m \times m$ and $U \subset D$ is a neighborhood 0 in $m$ for which $\pi \circ \exp =\pi \circ \psi$ is a diffeomorphism. Thus analogous to local Lie groups we have a local multiplication system $(U, F)$.

We now consider the Taylor's series for $F$ near the origin $\theta=$ $(0,0) \in m \times m$. Thus for $Z=(X, Y) \in m \times m$ and $Z^{k}=(Z, \cdots, Z)$ $k$-times we have for $t$ in a suitable interval $(-\delta, \delta) \subset R$ that

$$
F(t X, t Y)=F(\theta)+t F^{1}(\theta) Z^{1}+t^{2} / 2 F^{2}(\theta) Z^{2}+\cdots
$$

where $F^{k}(\theta)=D^{k} F(\theta)$ is the $k$ th derivative of $F$ at $\theta$ and is regarded as a symmetric $k$-linear function on $(m \times m)^{k}$ into $m$ [5]. In particular since $\mu(\bar{e}, \bar{e})=\bar{e}, F(\theta)=F(0,0)=0$. Next writing $Z=(X, 0)+(0$, $Y)$ we see that

$$
\begin{aligned}
D F(\theta) Z & =D F(\theta)[(X, 0)+(0, Y)] \\
& =[D F(\theta)](X, 0)+[D F(\theta)](0, Y) \\
& =P X+Q Y
\end{aligned}
$$

where $[D F(\theta)](X, 0)$ is regarded as a linear function of $X$ and denoted by $P X$ with $P$ an endomorphism of $m$ and similarly $[D F(\theta)](0, Y)=$ $Q Y$.

Using the symmetric bilinearity of $D^{2} F(\theta)$ we obtain for $Z=(X, Y)$

$$
\begin{aligned}
1 / 2\left[F^{2}(\theta)\right](Z, Z)= & 1 / 2 F^{2}(\theta)[(X, 0)+(0, Y), Z] \\
= & 1 / 2 F^{2}(\theta)[(X, 0),(X, 0)]+1 / 2 F^{2}(\theta)[(0, Y),(0, Y)] \\
& +F^{2}(\theta)[(X, 0),(0, Y)] .
\end{aligned}
$$


Now we note that for $X, Y \in m$

$$
\alpha(X, Y)=F^{2}(\theta)[(X, 0),(0, Y)]
$$

defines a bilinear function $\alpha$ on $m \times m$ into $m$ as follows. For $a \in R$ and $X, Y, Z \in m$

$$
\begin{aligned}
\alpha(a X+Z, Y) & =F^{2}(\theta)[(a X+Z, 0),(0, Y)] \\
& =F^{2}(\theta)[(a X, 0)+(Z, 0),(0, Y)] \\
& =F^{2}(\theta)[(a X, 0),(0, Y)]+F^{2}(\theta)[(Z, 0),(0, Y)] \\
& =a \alpha(X, Y)+\alpha(Z, Y)
\end{aligned}
$$

and similarly $\alpha$ is right-linear. Thus $m$ with the bilinear function $\alpha$ becomes a nonassociative algebra denoted by $(m, \alpha)$.

Note that the converse is true locally. Thus given a nonassociative algebra $(m, \alpha)$ we can find a neighborhood $D$ of 0 in $m$ so that $\mu(\pi \exp X, \pi \exp Y)=\pi \exp (X+Y+\alpha(X, Y))$ defines a local multiplicative system on some neighborhood $N^{*}$ of $\bar{e}$; this is analogous to formal Lie groups. Furthermore note that this multiplicative system has $\bar{e}$ as a two-sided identity and for $F(X, Y)=X+Y+\alpha(X, Y)$ we have $1 / 2 F^{2}(\theta)(X, Y)^{2}=\alpha(X, Y)$.

If the multiplicative system $(G / H, \mu)$ has $\bar{e}=e H$ as a right identity $(\mu(\bar{a}, \bar{e})=\bar{a})$, then in the above notation

$$
P=I \text { and } F^{k}(\theta)(X, 0)^{k}=0 .
$$

For if $t$ is in a suitable interval $(-\delta, \delta)$ of $R$ we have for $X \in m$ that

$$
\pi \exp t X=\mu(\pi \exp t X, \pi \exp 0)=\pi \exp F(t X, 0)
$$

and since $\pi \circ$ exp suitably restricted to $m$ is a diffeomorphism as previously discussed we have

$$
t X=F(t X, 0)=t P X+\frac{t^{2}}{2} F^{2}(\theta)(X, 0)^{2}+\cdots
$$

for $t$ in a suitable interval about 0 in $R$. Differentiating this formula at $t=0$ gives the results. A similar result holds if $(G / H, \mu)$ has $\bar{e}$ as a left identity. Thus if $(G / H, \mu)$ is an $H$-space; that is, $\bar{e}$ is a twosided identity, then $F(X, Y)=X+Y+\alpha(X, Y)+\cdots$.

3. Automorphisms. From the bijective correspondence between $G$-invariant affine connections on $G / H$ and nonassociative algebras $(m, \alpha)[6,8]$ we see that $\operatorname{Ad} H$ must be in the automorphism group, Aut $(m, \alpha)$, of the algebra $(m, \alpha)$. We shall show in this section that this condition is implied by $\tau(H) \subset \operatorname{Aut}(G / H, \mu)$; thus we want to consider multiplicative systems $(G / H, \mu)$ with $\tau(H) \subset \operatorname{Aut}(G / H, \mu)$. 
Definition. An analytic diffeomorphism $\eta: G / H \rightarrow G / H$ is an automorphism of $(G / H, \mu)$ if $\eta(\bar{e})=\bar{e}$ and $\eta \mu(\bar{x}, \bar{y})=\mu(\eta \bar{x}, \eta \bar{y})$ for all $\bar{x}, \bar{y} \in G / H$. We denote the set of such automorphisms by Aut $(G / H$, $\mu)$. An endomorphisms $S \in G L(m)$ is an automorphism of the algebra $(m, \alpha)$ if $S \alpha(X, Y)=\alpha(S X, S Y)$ for all $X, Y \in m$. We denote the set of such automorphisms by Aut $(m, \alpha)$.

For $\eta \in \operatorname{Aut}(G / H, \mu)$ and for $X \in m$ sufficiently near 0 in $m$ we can write

$$
\eta(\pi \exp X)=\pi \exp (\varphi(X))
$$

where $\varphi: m \rightarrow m$ is analytic at $0 \in m$ and $\varphi(0)=0$. Thus for $X, Y$ sufficiently near 0 in $m$ we can also write

$$
\eta \mu(\pi \exp X, \pi \exp Y)=\eta(\pi \exp F(X, Y))=\pi \exp (\varphi F(X, Y))
$$

and

$$
\begin{aligned}
\mu(\eta(\pi \exp X), \eta(\pi \exp Y)) & =\mu(\pi \exp (\varphi X), \pi \exp (\varphi Y)) \\
& =\pi \exp F(\varphi X, \varphi Y) .
\end{aligned}
$$

But since $\eta \in \operatorname{Aut}(G / H, \mu)$ we can conclude for $X, Y$ sufficiently near 0 in $m$

$$
\varphi F(X, Y)=F(\varphi X, \varphi Y)
$$

that is, $\varphi$ is an automorphism of a suitable local multiplicative system $(U, F)$.

We shall now expand $\varphi$ and $F$ in their Taylor's series to find conditions on $\eta \in \operatorname{Aut}(G / H, \mu)$ so that the differential $(T \eta)(\bar{e})$ is in Aut $(m, \alpha)$. First we note from (3.1) and the chain rule we have for $X \in m$

$$
\begin{aligned}
\operatorname{T\eta }(\bar{e})(X) & =[T(\pi \circ \exp \circ \varphi)(0)](X) \\
& =[T \pi(e) \circ T \exp (0) \circ T \varphi(0)](X) \\
& =T \varphi(0)(X) \\
& =\varphi^{1}(0)(X)
\end{aligned}
$$

because $T \exp (0)$ is the identity on $g$ and $T \pi(e)$ is the identity on $m$. From the Taylor's series

$$
F(X, Y)=P X+Q Y+\frac{F^{2}(\theta)}{2}(X, Y)^{2}+\cdots
$$

and

$$
\varphi(X)=\varphi^{1}(0) X+\frac{\varphi^{2}(0)}{2} X^{2}+\cdots
$$

we have for $X, Y$ sufficiently near 0 in $m$ 


$$
\begin{aligned}
\varphi(F(X, Y))= & \varphi^{1}(0) F(X, Y)+1 / 2 \varphi^{2}(0)(F(X, Y), F(X, Y))+\cdots \\
= & \varphi^{1}(0)\left(P X+Q Y+1 / 2 F^{2}(\theta)(X, Y)^{2}+\cdots\right) \\
& +1 / 2 \varphi^{2}(0)(P X+Q Y+\cdots, P X+Q Y+\cdots)+\cdots \\
= & \varphi^{1}(0) P X+\varphi^{1}(0) Q Y \\
& +1 / 2 \varphi^{1}(0) F^{2}(\theta)(X, Y)^{2}+1 / 2 \varphi^{2}(0)(P X+Q Y, P X+Q Y) \\
& +\varepsilon_{3}
\end{aligned}
$$

where $\varepsilon_{3}$ is of order three. Also

$$
\begin{aligned}
F(\varphi X, \varphi Y)= & P \varphi(X)+Q \varphi(Y)+1 / 2 F^{2}(\theta)(\varphi X, \varphi Y)^{2}+\cdots \\
= & P\left(\varphi^{1}(0) X+1 / 2 \varphi^{2}(0) X^{2}+\cdots\right)+Q\left(\varphi^{1}(0) Y+1 / 2 \varphi^{2}(0) Y^{2}\right. \\
& +\cdots) \\
& +1 / 2 F^{2}(\theta)\left(\varphi^{1}(0) X+\cdots, \varphi^{1}(0) Y+\cdots\right)^{2}+\cdots \\
= & P \varphi^{1}(0) X+Q \varphi^{1}(0) Y+1 / 2 P \varphi^{2}(0) X^{2}+1 / 2 Q \varphi^{2}(0) Y^{2} \\
& +1 / 2 F^{2}(\theta)\left(\varphi^{1}(0) X, \varphi^{1}(0) Y\right)^{2}+\varepsilon_{3} .
\end{aligned}
$$

Since $\varphi F(X, Y)=F(\varphi X, \varphi Y)$ we compare terms of the same degree to obtain

$$
\left[\varphi^{1}(0), P\right]=\left[\varphi^{1}(0), Q\right]=0
$$

and

$$
\begin{aligned}
1 / 2 \varphi^{1}(0) F^{2}(\theta)(X, Y)^{2} & +1 / 2 \varphi^{2}(0)(P X+Q Y, P X+Q Y) \\
= & 1 / 2 P \varphi^{2}(0) X^{2}+1 / 2 Q \varphi^{2} Y^{2} \\
& +1 / 2 F^{2}(\theta)\left(\varphi^{1}(0) X, \varphi^{1}(0) Y\right)^{2} .
\end{aligned}
$$

From (2.1) and this last equation we obtain by considering the expressions in both $X$ and $Y$ (i.e. replacing $X$ by $s X$ and $Y$ by $t Y$ ):

$$
\begin{gathered}
\varphi^{1}(0) F^{2}(\theta)[(X, 0),(0, Y)]-F^{2}(\theta)\left[\left(\varphi^{1}(0) X, 0\right),\left(0, \varphi^{1}(0) Y\right)\right] \\
=-\varphi^{2}(0)(P X, Q Y) .
\end{gathered}
$$

Recalling $\alpha(X, Y)=F^{2}(\theta)[(X, 0)(0, Y)]$ and equation $\left({ }^{* *}\right)$ and by definition $T \eta(\bar{e})$ is nonsingular we obtain the following.

LeMma 3.1. Let $(G / H, \mu)$ be a multiplicative system given locally by $\mu(\pi \exp X, \pi \exp Y)=\pi \exp F(X, Y)$ where $F(X, Y)=P X+Q Y+$ $1 / 2 F^{2}(\theta)(X, Y)^{2}+\cdots$ and let $\eta \in \operatorname{Aut}(G / H, \mu)$ given locally by $\eta(\pi \exp X)=$ $\pi \exp (\varphi(X))$. Then

(1) $[P, T \eta(\bar{e})]=[Q, T \eta(\bar{e})]=0$

(2) $T \eta(\bar{e}) \in$ Aut $(m, \alpha)$ if and only if $\varphi^{2}(0)(P X, Q Y)=0$ for all $X, Y \in m$.

Now for $u \in H$ and for $x \in G$ with $\pi x=x H \in G / H$ we have 


$$
\tau(u) \pi(x)=u x H=u x u^{-1}(u H)=\pi \sigma(u)(x)
$$

where $\sigma(u): G \rightarrow G: x \rightarrow u x u^{-1}$ is the inner automorphism of the group $G$ defined by $u$. Next recall [1] that $[T \sigma(u)](e)=\mathrm{Ad} u$ and for an automorphism $\sigma$ of $G$ that $\sigma(\exp X)=\exp (T \sigma(e) X)$. So we assume for $u \in H$ that $\eta=\tau(u) \in \operatorname{Aut}(G / H, \mu)$. Then the local representation gives

$$
\begin{aligned}
\pi \exp (\varphi(X)) & =\eta(\pi \exp X) \\
& =(\tau(u) \circ \pi)(\exp X) \\
& =\pi \circ \sigma(u)(\exp X) \\
& =\exp \pi([T \sigma(u)(e)](X)) \\
& =\pi \exp (\operatorname{Ad} u(X)) .
\end{aligned}
$$

Since $(G, H)$ is a reductive pair we have $\operatorname{Ad} H(m) \subset m$ and consequently for all $X$ in a suitable neighborhood of 0 in $m$ we have

$$
\varphi(X)=\operatorname{Ad} u(X) \text {. }
$$

Thus since $\varphi=\operatorname{Ad} u$ is linear we have from this equation, and $\left(^{* *}\right)$ applied to $\eta=\tau(u)$ that

$$
T \tau(u)(\bar{e})=T \varphi(0)=\operatorname{Ad} u .
$$

Since $\varphi=\operatorname{Ad} u$ is linear, its second derivative is zero; that is, $\varphi^{2}(0)=0$. This and Lemma 3.1 yield the following.

Proposition 3.2. Let $(G / H, \mu)$ be a multiplicative system so that $\tau(H) \subset \operatorname{Aut}(G / H, \mu)$. Let $\mu$ be given locally by $\mu(\pi \exp X, \pi \exp Y)=$ $\pi \exp F(X, Y)$ where $F(X, Y)=P X+Q Y+1 / 2 F^{2}(\theta)(X, Y)^{2}+\cdots$ and let $(m, \alpha)$ be the algebra determined by $F^{2}(\theta)$. Then

(1) $[P, \operatorname{Ad} u]=[Q, \operatorname{Ad} u]=0$ all $u \in H$.

(2) Ad $H \subset$ Aut $(m, \alpha)$.

(3) The algebra $(m, \alpha)$ defines a G-invariant affine connection on $G / H$.

4. Invariant multiplications. Let $(G / H, \mu)$ be a multiplicative system defined on the reductive space $G / H$ and let $g=m+h$ be the corresponding fixed decomposition. For $X \in m$ and for $T(G / H)$ the tangent bundle of $G / H$ define functions

$$
\iota(\mu, X): G / H \longrightarrow T(G / H): \bar{a} \longrightarrow[(T \mu)(\bar{a}, \bar{e})](0, X)
$$

and

$$
\imath(\mu, X): G / H \longrightarrow T(G / H): \bar{a} \longrightarrow[(T \mu)(\bar{e}, \bar{a})](X, 0)
$$


where $T \mu$ is the differential of the function $\mu: G / H \times G / H \rightarrow G / H$ which is evaluated, for example, at $(\bar{a}, \bar{e})$ and acting on tangent vectors $(0, X) \in T(G / H, \bar{a}) \times T(G / H, \bar{e})$.

Next note $\ell(\mu X)(\operatorname{resp} . \imath(\mu, X))$ is a vector field if and only if $\mu(\bar{a}, \bar{e})=\bar{a}(\operatorname{resp} . \mu(\bar{e}, \bar{a})=\bar{a})$. For if $\ell(\mu, X)$ is a vector field and $\mu(\bar{a}, \bar{e})=\bar{b}$, then $\ell(\mu, X)(\bar{a}) \in T(G / H, \bar{b})$ which is the tangent space of $G / H$ at $\bar{b}$. But $\ell(\mu, X)$ being a vector field means

$$
\bar{a}=i d y(\bar{a})=p \circ \measuredangle(\mu, X)(\bar{a})=\bar{b}
$$

where $p: T(G / H) \rightarrow G / H$ is the corresponding projection map. Conversely $\mu(\bar{a}, \bar{e})=\bar{a}$ easily implies $p \circ \ell(\mu, X)=i d y$; that is, $\ell(\mu, X)$ is a vector field. Similarly for $\_(\mu, X)$.

Also it is not difficult to see that in this case $\ell(\mu, X)$ and $s(\mu, X)$ are analytic vector fields which depend linearly on the parameter $X$. Since the results for $\iota(\mu, X)$ are similar to those for $\ell(\mu, X)$, we restrict ourselves to $\ell(\mu, X)$.

We now define the concept of an invariant multiplication which reduces to the familiar notion in Lie groups. Recall [4] if $\widetilde{X}: M \rightarrow$ $T(M)$ is an analytic vector field on a manifold $M$ where $T(M)$ is the tangent bundle over $M$ and if $f: M \rightarrow M$ is an analytic diffeomorphism, then $\widetilde{X}$ is $f$-invariant if

$$
T f(p)(\widetilde{X}(p))=\widetilde{X}(f(p))
$$

for all $p \in M$ where $T f(p): T(M, p) \rightarrow T(M, f(p))$ is the differential of $f$ at $p$.

Definition. Let $(G, H)$ be a reductive pair with $g=h+m$ the corresponding decomposition for $g$ and let $G / H$ be the homogeneous space of left cosets. Let $(G / H, \mu)$ be a multiplicative system with $\tau(H) \subset$ Aut $(G / H, \mu)$ and let $\Gamma=\left\{\tau(\exp A): A \in O_{m}\right\}$ where $O_{m}$ is a neighborhood of 0 in $m$ on which exp is one-to-one and $\left(\exp O_{m}\right) \cap H=\{e\} ;[1, \mathrm{p} .113]$. Let $\bar{e}=e H$ be a right identity for $(G / H, \mu)$; that is, $\mu(\bar{a}, \bar{e})=\bar{a}$, then $\mu$ is called $\Gamma$-invariant if for all $X \in m$ the vector fields $\ell(\mu, X)$ are invariant relative to the functions in $\Gamma$ as follows:

$$
T \tau(\exp A)(\bar{e}) \cdot \ell(\mu, X)(\bar{e})=\ell(\mu, X)(\tau(\exp A) \bar{e})
$$

for all $A$ in $O_{m}$.

REMARK. (1) Before considering the general case we first consider the system $(G, \mu)$. In this case $\Gamma$ can be replaced by all of $L(G)=$ $\{L(\alpha): a \in G\}$ where $L(\alpha): G \rightarrow G: x \rightarrow a x$, the multiplication in the group $G$. In particular we see that if $e$ is a right identity of $(G, \mu)$, then the vector field $\ell(X)$ is $\Gamma$-invariant if and only if $T L(a)(p) \cdot \iota(\mu, X)(p)=$ $\ell(\mu, X)(L(a) p)$; that is, the $\Gamma$-invariance at $e$ is actually global. Also 
it should be noted that when $\mu$ is the Lie group multiplication in $G$, then the $L(G)$-invariant vector field $\ell(X)$ equals the usual left $G$ invariant $\widetilde{X}$; note remark (3) below.

ExAmple 1. Let $f: G \rightarrow G$ be an analytic function on the Lie group $G$ so that $f(e)=e$, then the multiplication

$$
\mu(x, y)=m(x, f(y))=x f(y)
$$

is $L(G)$-invariant where $m$ is the Lie group multiplication in $G$. First $\mu(x, e)=x f(e)=x$ for all $x \in G$ so that $\iota(\mu, X)$ is a vector field on $G$. From remark (1) we have $m$ is a $L(G)$-invariant multiplication so that

$$
[(T m)(a, e)](0, U)=\ell(m, U)(a)=T L(a)(e)[(T m)(e, e) \cdot(0, U)] .
$$

Thus noting $\mu(x, y)=[m \circ(i d y \times f)](x, y)$ we have using the chain rule

$$
\begin{aligned}
\iota(\mu, X)(a) & =[(T \mu)(a, e)](0, X) \\
& =[T(m \circ(i d y \times f))(a, e)](0, X) \\
& =[T m(a, e) \circ(T i d y(a) \times T f(e))](0, X) \\
& =[T m(a, e)](T i d y(a) \cdot 0, T f(e) \cdot X) \\
& =[T m(a, e)](0, T f(e) \cdot X) \\
& =T L(a)(e)[T m(e, e) \cdot(T i d y(e) \cdot 0, T f(e) \cdot X)] \\
& =T L(a)(e)[T(m \circ(i d y \times f))(e, e) \cdot(0, X)] \\
& =T L(a)(e)[(T \mu)(e, e) \cdot(0, X)] \\
& =T L(a)(e) \measuredangle(\mu, X)(e)
\end{aligned}
$$

so that $\mu$ is left $L(G)$-invariant. Other examples can easily be constructed where the multiplication need not have the "separation of variable property". Thus locally an $L(\exp g)$-invariant multiplication $\mu$ can be given by $\mu(\exp X, \exp Y)=\exp F(X, Y)$ where

$$
F(X, Y)=C(X, f(X, Y))
$$

with $f: g \times g \rightarrow g$ analytic at $(0,0)$, and $f(X, 0)=0$, and $C(X, Y)=$ $X+Y+1 / 2[X Y]+\cdots$ is the Campbell-Hausdorff formula. We leave it as an open problem to see if this is the most general way of obtaining the local expression for an $L(G)$-invariant multiplication on $G$.

REmarks. We shall soon give the local formula for a $\Gamma$-invariant multiplication on $G / H$. But first we give a few remarks and formulas.

(2) If $(G / H, \mu)$ is a Lie group; i.e. $H$ normal, then since $\tau(a) \bar{x}=$ axH we have $\mu(\bar{a}, \bar{x})=L(\bar{a}) \bar{x}$. Thus locally $\mu$ is $L(G / H)$-invariant (as a group) if and only if $\mu$ is $\Gamma$-invariant (as a homogeneous space). 
Also when $H$ is a normal subgroup with $\mu(\bar{a}, \bar{b})=a b H$, then $\tau(H) \subset$ Aut $(G / H, \mu)$ because for $u \in H$ we have

$$
\begin{aligned}
\tau(u) \mu(\bar{a}, \bar{b}) & =u a b H \\
& =\left(u a u^{-1} u b u^{-1}\right) H \\
& =u a u^{-1} H \cdot u b u^{-1} H \\
& =\mu(\tau(u) \bar{a}, \tau(u) \bar{b}) .
\end{aligned}
$$

(3) Let $(G / H, \mu)$ be a multiplicative system with $\mu$ given locally by

$$
F(X, Y)=X+Q Y+\alpha(X, Y)+1 / 2 F^{2}(\theta)(0, Y)^{2}+\cdots
$$

as in $\S 2$, where $\theta=(0,0)$ and $\alpha(X, Y)=F^{2}(\theta)[(X, 0),(0, Y)]$. Then the vector field $\ell(\mu, X)$ satisfies

$$
\ell(\mu, X)(\bar{e})=Q X .
$$

For from $\mu(\pi \circ \exp X, \pi \circ \exp Y)=\pi \circ \exp F(X, Y)$ we obtain $\mu \circ(\pi \circ \exp \times$ $\pi \circ \exp )=\pi \circ \exp \circ F$. Using $T(\pi \circ \exp )(0)=i d y$ on $m$ and the chain rule we obtain

$$
\begin{aligned}
\iota(\mu, X)(\bar{e}) & =[(T \mu)(\bar{e}, \bar{e})](0, X) \\
& =T[(\pi \circ \exp \circ F)(0,0)](0, X) \\
& =T(\pi \circ \exp )(0)(0+Q X) \\
& =Q X
\end{aligned}
$$

recalling $[(T F)(0,0)](U, V)=U+Q V$.

(4) We must restrict ourselves to the set $\Gamma=\{\tau(\exp A): A \epsilon$ $\left.O_{m}\right\}$ and not the group generated by $\tau\left(\exp O_{m}\right)$ because this group is frequently $G$ since $m$ frequently generates $g$. For example, if $g$ is simple, then $m+[m m]$ is an ideal of $g$ and therefore equals $g$.

However if for the system $(G / H, \mu)$ we have $\mu$ is $\tau(G)$-invariant, then it is $\tau(H)$-invariant. This with $\tau(H) \subset$ Aut $(G / H, \mu)$ yield the following computations which indicate that $\tau(G)$-invariance is too strong of a condition. With $\mu$ given by $F(X, Y)=X+Q Y+\cdots$ as in remark (3) we see from Proposition 3.2 that $\tau(H) \subset(G / H, \mu)$ implies [Ad $u, Q]=0$ for all $u \in H$. From $\mu$ being $\tau(H)$ invariant we have

$$
T \tau(u)(\bar{e}) \cdot \ell(\mu, X)(\bar{e})=\ell(\mu, X)(\tau(u) \bar{e})=\ell(\mu, X)(\bar{e}) .
$$

But from formula (3.2) we have $T \tau(u)(\bar{e})=\operatorname{Ad} u$ and from remark (3) we have $\ell(\mu, X)(\bar{e})=Q X$; thus

$$
(\text { Ad } u)(Q X)=Q X \text {. }
$$

This gives, since $[\operatorname{Ad} u, Q]=0$ that $Q(\operatorname{Ad} u-I) X=0$ for all $u \in H$ and $X \in m$. Thus for $u=\exp U$ with $U \in h$ and using $\operatorname{Ad}(\exp U)=e^{\operatorname{ad} U}$ 
we obtain

$$
0=(Q \circ \operatorname{ad} U)(X)=(\operatorname{ad} U \circ Q)(X) .
$$

If $Q$ is nonsingular we obtain $(\operatorname{ad} h)(m)=0$ so that $h$ is an ideal in $g$; this is usually not the case.

We now obtain sufficient information concerning the multiplication $\mu$ from the Taylor's series for $F(X, Y)$; note the converse statement in Proposition 4.7.

THEOREM 4.6. Let $(G, H)$ be a reductive pair with $(G / H, \mu)$ a multiplicative system with $\tau(H) \subset$ Aut $(G / H, \mu)$. Let $\mu$ be $\Gamma$-invariant and for $X, Y \in m$ in a suitable neighborhood of 0 in $m$ let $\mu$ be given locally by $\mu(\pi \exp X, \pi \exp Y)=\pi \exp F(X, Y)$ where $F$ is given by the Taylor's series

$$
\begin{gathered}
F(X, Y)=X+Q Y+\alpha(X, Y)+1 / 2 F^{2}(\theta)(0, Y)^{2} \\
+\sum_{n=3}^{\infty} 1 / n ! F^{n}(\theta)(X, Y)^{n}
\end{gathered}
$$

Then

(1) $\alpha(X, Y)=1 / 2 X \cdot Q Y\left(\equiv 1 / 2[X Q Y]_{m}\right)$

(2) If $\pi^{\prime}=T \pi(e)$ and if

$$
F_{n} \equiv F_{n}(U, V)=F^{n}(\theta)[(U, 0), \cdots,(U, 0),(0, V)]
$$

where $(U, 0)$ occurs $(n-1)$-times and $n>2$, then for $F_{1} \equiv F_{1}(X, Y)=$ $Q Y$ we have

$$
\begin{aligned}
0= & \pi^{\prime}\left[\mu(n, 0) F_{n}+\mu(n, 1) \operatorname{ad} X\left(F_{n-1}\right)+\cdots\right. \\
& \left.+\mu(n, k)(\operatorname{ad} X)^{k}\left(F_{n-k}\right)+\cdots+\mu(n, n-1)(\operatorname{ad} X)^{n-1} F_{1}\right]
\end{aligned}
$$

where $\mu(n, k)=(-1)^{k} /(k+1) !(n-k-1)$ !. Thus we have an iterative formula for part of the Taylor's series for $F$ which is the best possible obtainable from the $\Gamma$-invariance condition.

(3) For each $u \in H$ and $n=2,3, \ldots$

$$
(\operatorname{Ad} u) \cdot F^{n}(\theta)(X, Y)^{n}=F^{n}(\theta)(\operatorname{Ad} u \cdot X, \operatorname{Ad} u \cdot Y)^{n} \text {. }
$$

In particular $(\operatorname{Ad} u) \cdot F_{n}(X, Y)=F_{n}(\operatorname{Ad} u \cdot X, \operatorname{Ad} u, Y)$.

Proof. We have $\tau(a) \circ \pi=\pi \circ L(a)$ and from remark (3)

$$
[\mu \circ(\pi \times \pi) \circ(\exp \times \exp )](X, Y)=[\pi \circ \exp \circ F](X, Y) .
$$

Using this equation and the chain rule we obtain for $\bar{a}=\pi \exp X$ and 
$\bar{e}=\pi \exp 0$ that

$$
[(T \mu)(\bar{a}, \bar{e})](0, Y)=[T(\pi \circ \exp \circ F)(X, 0)](0, Y)
$$

which is used in the fifth equality below.

From [1, p. 95] we have for $A$ in a suitable neighborhood of 0 in $g$ that

$$
T[L(\exp (-A)) \circ \exp ](A)=\frac{I-e^{-\mathrm{ad} A}}{\operatorname{ad} A}
$$

where $\left(I-e^{-P}\right) / P=\sum_{k=0}^{\infty}(-P)^{k} /(k+1)$ !. Also from $\tau\left(a^{-1}\right) \circ \tau(a)=i d y$ on $G / H$ we obtain

$$
T \tau\left(a^{-1}\right)(\bar{a}) \circ T \tau(a)(\bar{e})=I
$$

which gives the inverse for $T \tau(a)(\bar{e})$.

We now use the above formulas and the chain rule to obtain for $X, Y$ in a suitable neighborhood of 0 in $m$ and $a=\exp X$,

$$
\begin{aligned}
Q Y & =\iota(\mu, Y)(\bar{e}) \\
& =[T \tau(a)(\bar{e})]^{-1} \iota(\mu, Y)(\bar{a}) \\
& =T \tau\left(a^{-1}\right)(\bar{a}) \cdot[(T \mu)(\bar{a}, \bar{e})](0, Y) \\
& =\left[T\left(\tau\left(a^{-1}\right) \circ \mu\right)(\bar{a}, \bar{e})\right](0, Y) \\
& =\left[T\left(\tau\left(a^{-1}\right) \circ \pi \circ \exp \circ F\right)(X, 0)\right](0, Y) \\
& =T \pi(e) \cdot T(L(\exp (-X)) \circ \exp )(X) \cdot[(T F)(X, 0)](0, Y) \\
& =T \pi(e) \cdot \frac{I-e^{-\mathrm{ad} X}}{\operatorname{ad} X} \cdot[(T F)(X, 0)](0, Y)
\end{aligned}
$$

where

$$
\begin{aligned}
F^{\prime} & \equiv[(T F)(X, 0)](0 Y) \\
& =\lim _{t \rightarrow 0} \frac{1}{t}[F(X, t Y)-F(X, 0)] \\
& =\lim _{t \rightarrow 0} \frac{1}{t}\left[X+t Q Y+t \alpha(X, Y)+t^{2} / 2 F^{2}(\theta)(0, Y)^{2}+\cdots-X\right] \\
& =Q Y+\alpha(X, Y)+\sum_{n=3}^{\infty} \frac{1}{(n-1) !} F_{n}(X, Y) .
\end{aligned}
$$

To see this last equality just note that by induction we have

$$
\begin{aligned}
F^{n}(\theta)(X, t Y)^{n} & =F^{n}(\theta)(X, 0)^{n}+n t F_{n}(X, Y)+o\left(t^{2}\right) \\
& =n t F_{n}(X, Y)+o\left(t^{2}\right)
\end{aligned}
$$

using $F^{n}(\theta)(X, 0)^{n}=0$ since $(G / H, \mu)$ has $\bar{e}$ as a right identity.

From the series for $\left(I-e^{-\mathrm{ad} X}\right) / \mathrm{ad} X$ we obtain 


$$
\begin{aligned}
Q Y= & \pi^{\prime} \sum_{k=0}^{\infty}\left(\frac{(-\operatorname{ad} X)^{k}}{(k+1) !}\right)\left(F^{\prime}\right) \\
= & \pi^{\prime}\left[I F^{\prime}-\frac{\operatorname{ad} X}{2 !} F^{\prime}+\cdots+\frac{(-\operatorname{ad} X)^{k}}{(k+1) !} F^{\prime}+\cdots\right] \\
= & \pi^{\prime}\left[Q Y+\alpha(X, Y)+\sum_{n=3}^{\infty} F_{n} /(n-1) !\right. \\
- & 1 / 2(\operatorname{ad} X)\left(Q Y+\alpha(X, Y)+\sum_{n=3}^{\infty} F_{n} /(n-1) !\right) \\
& \left.+\cdots+\frac{(-\operatorname{ad} X)^{k}}{(k+1) !}\left(Q Y+\alpha(X, Y)+\sum_{n=3}^{\infty} F_{n} /(n-1) !\right)+\cdots\right] \\
= & \pi^{\prime}[Q Y+\alpha(X, Y)-1 / 2(\operatorname{ad} X)(Q Y)+\cdots] \\
= & \pi^{\prime} Q Y+\pi^{\prime} \alpha(X, Y)-\frac{\pi^{\prime}}{2}(\operatorname{ad} X)(Q Y)+\cdots
\end{aligned}
$$

Thus since $Q Y$ and $\alpha(X, Y)$ are in $m$ and $\pi^{\prime} \mid m$ is the identity, we obtain

$$
\begin{aligned}
\alpha(X, Y) & =\frac{\pi^{\prime}}{2}(\operatorname{ad} X)(Q Y) \\
& =\frac{\pi^{\prime}}{2}\left(X \cdot Q Y+[X Q Y]_{h}\right) \\
& =\frac{1}{2} X \cdot Q Y .
\end{aligned}
$$

(Recall $[U V]_{h}$ is the component of [UV] which is in $h$ ). Similarly by noting $F_{k}(X, Y)$ is homogeneous in $X$ of degree $k-1$ we combine those terms of degree $n-1$ in $X$ to obtain

$$
\begin{aligned}
0= & \pi^{\prime}\left[\frac{F_{n}}{(n-1) !}-\frac{1}{2}(\operatorname{ad} X) \frac{F_{n-1}}{(n-2 !)}+\cdots+\frac{(-1)^{k}(\operatorname{ad} X)^{k} F_{n-k}}{(k+1) !(n-k-1) !}\right. \\
& \left.+\cdots+\frac{(-1)^{n-1}}{n !}(\operatorname{ad} X)^{n-1} F_{1}\right]
\end{aligned}
$$

where $F_{1}=Q Y$.

Equation (3) in the theorem follows from Proposition 3.2 and the remarks preceding it. Thus for $\eta \in \operatorname{Aut}(G / H, \mu)$ we wrote locally $\eta(\pi \exp X)=\pi \exp (\dot{\phi}(X))$ and showed $\phi F(X, Y)=F(\phi X, \phi Y)$. In particular for $\eta=\tau(u)$ we showed $\phi=\operatorname{Ad} u$ for $u \in H$ so that from the Taylor's series for $F$ and the linearity of $\phi=\operatorname{Ad} u$ we obtain (3).

EXAMPLE 2. These formulas can also be used to construct examples locally. Thus let $G$ be nilpotent so that ad $X$ is nilpotent for all $X \in g$; that is, there exists $n$ so that for all $X \in g,(\operatorname{ad} X)^{n}=0$. Let the function $F_{l}$ be given by the iteration formulas: $F_{0}=$ 
$X, F_{1}(X, Y)=Q Y, 0=\pi^{\prime}\left[\mu(2,0) F_{2}+\mu(2,1)\right.$ ad $\left.X F_{1}\right]$, etc. Set $K(X, Y)=$ $\sum_{k=0}^{\infty} F_{k}(X, Y) / k$ ! which is a finite sum by nilpotency of $G$. Note that $K$ is analytic and for the $k$-th derivative $K^{k}(\theta)(X, Y)^{k}=F_{k}(X, Y) \equiv$ $K_{k}(X, Y)$. Thus $K$ has the above Taylor's series and we can define locally $\mu(\pi \exp X, \pi \exp Y)=\pi \exp K(X, Y)$. This is (locally) $\Gamma$-invariant because $K$, in terms of its Taylor's series, satisfies the iteration equations of the theorem and the process of the proof is reversable.

Next by induction using the iteration equation we also obtain $\phi F_{k}(X, Y)=F_{k}(\phi X, \phi Y)$ for $\phi=\mathrm{Ad} u$ with $u \in H$. This uses Proposition 3.2 as follows: $\phi F_{1}(X, Y)=\operatorname{Ad} u Q Y=Q(\operatorname{Ad} u Y)=F_{1}(X, \phi Y)=F(\phi X$, $\phi Y)$ since " $X$ " does not occur in the formula for $F_{1}$. Also reductivity of the pair $(G, H)$ is used to commute $\pi^{\prime}$ and $\operatorname{Ad} u$ : for $Y \in m,\left(\pi^{\prime} \circ \operatorname{Ad} u\right)(Y)=$ $\operatorname{Ad} u(Y)=\left(\operatorname{Ad} u \circ \pi^{\prime}\right)(Y)$. Thus since $K(X, Y)=\sum F_{k}(X, Y) / k !$ we have $\phi K(X, Y)=K(\phi X, \phi Y)$ so that using the results of $\S 3$ and the definition of $\phi$ by $\tau(u)(\pi \exp X)=\pi \exp (\phi X)$ we have locally $\tau(H) \subset \operatorname{Aut}(G / H, \mu)$ as follows:

$$
\begin{aligned}
\tau(u) \mu(\pi \exp X, \pi \exp Y) & =\tau(u)(\pi \exp K(X, Y)) \\
& =\pi \exp (\phi K(X, Y)) \\
& =\pi \exp K(\phi X, \phi Y)
\end{aligned}
$$

and

$$
\begin{aligned}
\mu(\tau(u)(\pi \exp X), \tau(u)(\pi \exp Y)) & =\mu(\pi \exp (\phi Y), \pi \exp (\phi Y)) \\
& =\pi \exp K(\phi X, \phi Y) .
\end{aligned}
$$

We extend the above notions in the following result to obtain a converse to Theorem 4.6.

Proposition 4.7. Let $(G, H)$ be a reductive pair with fixed decomposition $g=m+h$. Let $F_{k}: m \times m \rightarrow m$ for $k=1,2, \cdots$ be a sequence of multilinear functions which satisfy the iterative equation (2) and equation (3) of Theorem 4.6; that is, for all $u \in H,(\operatorname{Ad} u) \cdot F_{k}(X, Y)=$ $F_{k}(\operatorname{Ad} u \cdot X, \operatorname{Ad} u \cdot Y)$. Then for all $X, Y$ in a suitable neighborhood of 0 in $m$ the series $X+\sum_{k=1}^{\infty} 1 / k ! F_{k}(X, Y)$ converges absolutely and uniformly to a function $K(X, Y)$ which is analytic at $\theta=(0,0) \in m \times$ $m$ and the multiplication $\mu(\pi \exp X, \pi \exp Y)=\pi \exp K(X, Y)$ defines a local multiplicative system $(G / H, \mu)$ so that $\mu$ is locally $\Gamma$-invariant.

Proof. Using the obvious extension of the results in example (2) above, it suffices to prove the series converges to an analytic function $K$ so that the derivatives $K^{k}(\theta)(X, Y)^{k}=F_{k}(X, Y)$. To show that the series converges absolutely and uniformally for $X, Y$ in a suitable neighborhood of 0 in $m$, we let $B_{k}=F_{k+1} / k$ ! for $k=1,2, \cdots$ and let $S=\pi^{\prime}$ and $T=\operatorname{ad} X$. Then from the iteration formula we 
obtain $F_{1}=S Q Y$ and

$$
\begin{aligned}
B_{1} & =\frac{S T}{2 !} F_{1} \\
B_{2} & =\frac{S T}{2 !} B_{1}-\frac{S T^{2}}{3 !} F_{1} \\
B_{3} & =\frac{S T}{2 !} B_{2}-\frac{S T^{2}}{3 !} B_{1}+\frac{S T^{3}}{4 !} F_{1} \\
& \vdots \\
B_{n} & =\frac{S T}{2 !} B_{n-1}-\frac{S T^{2}}{3 !} B_{n-2}+\cdots+\frac{(-1)^{n+1}}{(n+1) !} S T^{n} F_{1} .
\end{aligned}
$$

Now let || || denote either the operator or the Euclidean norm, then we have $\|S\|=1$. Let $r=1$ and let $c$ be a fixed number with $c>$ $5 e$ where $e=2.71 \cdots$. Then for $F_{1}=S Q Y$ with $X \in \mathscr{A} \equiv\{X \in m$ : $\|$ ad $X \|<r\}$ and $Y \in \mathscr{B} \equiv\{Y \in m:\|Q Y\|<1\}$ we have $\|T\|=\|\operatorname{ad} X\|<$ $r=1$ and $\left\|B_{1}\right\|=1 / 2\left\|S T F_{1}\right\| \leqq 1 / 2\|S\| \cdot\|T\| \cdot\left\|F_{1}\right\|<1 / 2<c$. Assume for all $k<n$ that $\sum_{i=1}^{k}\left\|B_{i}\right\|<c$, then for $k=n$ we have from the equations for the $B$ 's

$$
\begin{aligned}
\sum_{i=1}^{n}\left\|B_{i}\right\|= & \left\|\frac{S T}{2 !} F_{1}\right\| \\
& +\left\|\frac{S T}{2 !} B_{1}-\frac{S T^{2}}{3 !} F_{1}\right\| \\
& +\left\|\frac{S T}{2 !} B_{2}-\frac{S T^{2}}{3 !} B_{1}+\frac{S T^{3}}{4 !} F_{1}\right\|+\cdots \\
& +\left\|\frac{S T}{2 !} B_{n-1}-\frac{S T^{2}}{3 !} B_{n-2}+\cdots+\frac{(-1)^{n+1}}{(n+1) !} S T^{n} F_{1}\right\| \\
\leqq & \|S\|\left(\frac{\|T\|}{2 !}+\frac{\left\|T^{2}\right\|}{3 !}+\cdots+\frac{\left\|T^{n}\right\|}{(n+1) !}\right)\left\|F_{1}\right\| \\
& +\frac{\|S T\|}{2 !}\left(\sum_{i=1}^{n-1}\left\|B_{i}\right\|\right)+\frac{\left\|S T^{2}\right\|}{3 !}\left(\sum_{i=1}^{n-2}\left\|B_{i}\right\|\right) \\
& +\cdots+\frac{\left\|S T^{n-1}\right\|}{n !}\left\|B_{1}\right\| \\
\leqq & \left(\frac{r}{2 !}+\frac{r^{2}}{3 !}+\cdots+\frac{r^{n}}{(n+1) !}\right) \\
& +\frac{r}{2 !} c+\frac{r^{2}}{3 !} c+\cdots+\frac{r^{n-1}}{n !} c
\end{aligned}
$$

using the induction hypothesis and $\|T\|<r$. But if $d(k, r)=\sum_{i=1}^{k} r^{i} /(i+1)$ !, then 


$$
r d(k, r)+1+r=1+r+\frac{r^{2}}{2 !}+\cdots+\frac{r^{k+1}}{(k+1) !}<e^{r}=e
$$

since $r=1$. Thus $d(k, r)<(2.8-2) / 1=.8$. Therefore $\sum_{i=1}^{n}\left\|B_{i}\right\|<$ $.8+.8 c=.8(1+c)<c$ because $c>5 e$. Thus the series $\sum B_{k}(X, Y)$ converges absolutely for all $(X, Y)$ with $X \in \mathscr{A}$ and $Y \in \mathscr{B}$ to a function $\bar{k}(X, Y)$. Since $\left\|B_{k}\right\| / k+1<\left\|B_{k}\right\|$ we see that $\sum B_{k} /(k+1)$ converges absolutely to a function $k(X, Y)$.

This series converges uniformly on $\mathscr{A} \times \mathscr{B}$ to $k(X, Y)$ as follows. On $\mathscr{A} \times \mathscr{B}$ the partial sums $\sum_{k=1}^{n} B_{k}$ are bounded by $c>5 e$. Thus since $1 / k+1 \rightarrow 0$ we have from a standard result that the series $\sum B_{k} / k+1$ converges uniformly on $\mathscr{A} \times \mathscr{B}$. But $B_{k} / k+1=F_{k+1} /$ $(k+1)$ ! so that $X+\sum F_{k}(X, Y) / k$ ! converges uniformly on $\mathscr{A} \times \mathscr{B}$ to $K(X, Y)$. Using this we see from $[2, \S 3]$ that $K$ is analytic at $\theta=(0,0)$ and $K^{k}(\theta)(X, Y)^{k}=F_{k}(X, Y)$ as desired.

5. Connections and holonomy. From [6] there is a bijective correspondence between $G$-invariant connections on the reductive space $G / H$ and non-associative algebras $(m, \alpha)$ with $\operatorname{Ad} H \subset \operatorname{Aut}(m, \alpha)$. Thus if this algebra $(m, \alpha)$ is induced by a multiplicative system $(G / H, \mu)$ we obtain a connection "induced by $\mu$ " and we discuss such connections and the corresponding holonomy algebra (Lie algebra of the holonomy group). Thus for $X, Y, Z \in m$ let

$$
a(X): m \rightarrow m: Y \rightarrow \alpha(X, Y) \text { and } R(X, Y): m \rightarrow m: Z \rightarrow R(Y, Y) Z
$$

where $R(X, Y) Z=\alpha(X, \alpha(Y, Z))-\alpha(Y, \alpha(X, Z))-\alpha(X Y, Z)-[h(X$, $Y) Z$ ] is the curvature evaluated at $\bar{e}=e H$ in $G / H$ [6]; recall that $X Y=[X Y]_{m}\left(\operatorname{resp} . h(X, Y)=[X Y]_{h}\right)$ is the projection of $[X Y]$ in $g$ into $m($ resp. $h)$. From [7] the holonomy algebra, denoted by hol $(\alpha)$, is the smallest Lie algebra hol $(\alpha)$ of endomorphisms of $m$ so that $R(X$, $Y) \in \operatorname{hol}(\alpha)$ and $[a(X)$, hol $(\alpha)] \subset \operatorname{hol}(\alpha)$ for all $X, Y \in m$.

We shall say that the holonomy group acts irreducibly on $G / H$ in case hol $(\alpha)$ act irreducibly on $m$. This can be stated in terms of the algebra $(m, \alpha)$ as follows. A left ideal of the algebra $(m, \alpha)$ is a subspace $n$ of $m$ such that $\alpha(m, n) \subset n$. Thus from the formula for $R(X, Y) Z$ we see that a left ideal $n$ which is invariant under ad $h(m, m)=\{\operatorname{ad} h(X, Y): X, Y \in m\}$ is hol $(\alpha)$-invariant. Therefore the holonomy irreducibility of $G / H$ implies $(m, \alpha)$ has no left ideals which are $\operatorname{ad} h(m, m)$-invariant.

We now consider the connection of the first kind which is a well behaved, easy to construct connection. From [6] we see that on the reductive space $G / H$ there exists a unique $G$-invariant connection which has zero torsion tensor and such that a 1-parameter subgroup $x(t)=\exp t X$ of $G$ generated by $X \in m$ projects by $\pi: G \rightarrow G / H: x(t) \rightarrow$ 
$\bar{x}(t)$ into a geodesic $\bar{x}(t)$ in $G / H$. In this case $\alpha(X, Y)=1 / 2 X Y$ and the connection is called the connection of the first kind relative to a fixed decomposition $g=m+h$.

Thus since this multiplication $\alpha(X, Y)=1 / 2 X Y$ is anti-commutative, a left ideal is a two sided ideal; therefore holonomy irreducibility implies $(m, \alpha)$ has no ideals invariant under ad $h(m, m)$. But using the Jacobi identity, ad $h(m, m)$ is contained in the derivation algebra of $(m, \alpha)$ so that the algebra $(m, \alpha)$ must contain no proper ideals or $m m=0$; that is, the holonomy irreducibility implies $(m, \alpha)$ is the zero algebra or simple. This uses a result in [8] which states if a finite dimensional nonassociative algebra over $R$ which is not the zero algebra has a proper ideal, then it has a proper ideal invariant under its derivation algebra.

If the connection on $G / H$ induced by $(m, \alpha)$ is pseudo-Riemannian, then from [6] there exists a nondegenerate symmetric bilinear form $C: m \times m \rightarrow R$ satisfying

$$
C(\operatorname{ad} U \cdot X, Y)+C(X, \operatorname{ad} U \cdot Y)=0
$$

and

$$
C(a(Z) \cdot X, Y)+C(X, a(Z) \cdot Y)=0
$$

for all $X, Y, Z$ in $m$ and $U$ in $h$; that is, the endomorphisms ad $U$ and $a(Z)$ are $C$-skew symmetric. Also for this connection we have [6], $0=\operatorname{Tor}(X, Y)=\alpha(X, Y)-\alpha(X, Y)-X Y$ and the multiplication function $\alpha$ is determined by

$$
2 C(Z, \alpha(X, Y))=C(Z, X Y)+C(Z X, Y)+C(X, Z Y) .
$$

We shall denote the algebra $m$ with multiplication $\alpha(X, Y)=1 / 2 X Y$ by $(m, 1 / 2 X Y)$ and we shall denote the algebra $(m, \alpha)$ with a nondegenerate from $C$ inducing a pseudo-Riemannian connection (i.e. satisfying the above equations) by $(m, \alpha, C)$. In particular, if $C$ is positive definite so that it induces a Riemannian connection, then from the deRham decomposition [4] the original connection is built up from its irreducible components.

We next use the algebra $(m, \alpha)$ obtained from a multiplication to obtain a connection. Thus let $(G / H, \mu)$ be a multiplicative system as before and let

$$
\mu(\pi \exp X, \pi \exp Y)=\pi \exp F(X, Y)
$$

where we have

$$
F(X, Y)=P X+Q Y+\alpha(X, Y)+\cdots
$$

with $\alpha(X, Y)=F^{2}(\theta)[(X, 0),(0, Y)]$ a bilinear multiplication on $m$ so that $\operatorname{Ad} H \subset$ Aut $(m, \alpha)$. For a $\Gamma$-invariant multiplication we obtained 
in $\S 4$ (using the notation $X Y=X \cdot Y$ )

$$
\alpha(X, Y)=1 / 2 X \cdot Q Y
$$

thus if $L(X): m \rightarrow m: Y \rightarrow X Y$ we have for all $X \in m$ and $U \in h$ that

$$
a(X)=1 / 2 L(X) \circ Q \text { and }[\operatorname{ad} U, Q]=0
$$

using the results of $\S 3$.

LEMma 5.1. Let $(G / H, \mu)$ be a multiplicative system as above and let $\mu$ be $\Gamma$-invariant. Then the kernel of $Q$ is an ad $h$-invariant left ideal of $(m, \alpha)$.

Proof. Let $n=\operatorname{ker} Q$, then since $[\operatorname{ad} h, Q]=0$ we see that $n$ is ad $h$-invariant. Also $\alpha(m, n)=1 / 2 m \cdot Q n=0$ so that $\alpha(m, n) \subset n$; that is, $n$ is a left ideal.

Lemma 5.2. Let $(G / H, \mu)$ be a multiplicative system as before which induces a nonzero algebra $(m, \alpha)$ and a corresponding connection on $G / H$. Let $\mu$ be $\Gamma$-invariant and let hol $(\alpha)$ be irreducible, then $Q$ is nonsingular.

Proof. Suppose $\mu$ is $\Gamma$-invariant and hol $(\alpha)$ is irreducible. Then from the remarks at the beginning of this section, the algebra $(m, \alpha)$ has no left ideals which are ad $h(m, m)$-invariant. But from Lemma 5.1 , the kernel of $Q$ is such an ideal. Thus the kernel of $Q$ is zero since we are assuming $\alpha(X, Y)=1 / 2 X \cdot Q Y$ is not identically zero.

We use these lemmas in the next two results where we compare an irreducible connection induced by a multiplication with the irreducible connection of the first kind.

THEOREM 5.3. Let $(G, H)$ be a reductive pair so that for the decomposition $g=m+h$ we have $[m, m]_{m} \neq 0$. Let $(G / H, \mu)$ be a multiplicative system as before so that $\mu$ is $\Gamma$-invariant and let the connection induced by $\mu$ via the algebra $(m, \alpha)$ be a holonomy irreducible pseudo-Riemannian connection. If the algebra $(m, 1 / 2 X Y)$ is simple, then $\alpha(X, Y)=1 / 2 X Y$; thus the connection by $\mu$ is of the first kind.

Proof. First assume $\mu$ is $\Gamma$-invariant, then from $\alpha(X, Y)=$ $1 / 2 X \cdot Q Y$ and

$$
\begin{aligned}
0 & =\operatorname{Tor}(X, Y)=\alpha(X, Y)-\alpha(Y, X)-X Y \\
& =1 / 2 X \cdot Q Y-1 / 2 \cdot Q X-X Y
\end{aligned}
$$

we obtain 


$$
L(X) \circ Q=L((-2 I-Q) X) .
$$

Thus from $a(X) Y=\alpha(X, Y)$,

$$
2 a(X)=L(X) \circ Q=L((2 I-Q) X) .
$$

Next by Lemma 5.2 and the hypothesis of irreducibility we see that $Q$ is nonsingular. From this we obtain $2 I-Q$ is nonsingular as follows. If $(2 I-Q) A=0$, then using (5.3) $L(A) \circ Q=0$. But since $Q$ is nonsingular, $L(A)=0$. Thus if $A \neq 0$, this means that the one dimensional subspace $R A$ is an ideal in the simple algebra $(m, X Y)$; consequently $A=0$. Since $2 I-Q$ is nonsingular, $(2 I-Q) m=m$ and from formula (5.3) we obtain $a(m)=L(m)$. Next recall that the elements of $a(m)$ are $C$-skew so that the elements of $L(m)$ are also $C$-skew; thus

$$
C(Z X, Y)+C(X, Z Y)=0 \text {. }
$$

But from formula (5.1) which uniquely determines $\alpha$ in terms of $C$ and $(m, X Y)$ we obtain

$$
2 C(Z, \alpha(X, Y))=C(Z, X Y)
$$

that is, $\alpha(X, Y)=1 / 2 X Y$. Since $(m, X Y)$ is simple, this also implies $Q=I$.

COROLlaRY 5.4. Let the reductive pair $(G, H)$ and the multiplicative system $(G / H, \mu)$ be as in Theorem 5.3. If the corresponding Lie algebra $g$ is simple and $h$ is semi-simple and $g=m+h$ where $m=h^{\perp}$ the orthogonal complement relative to the Killing form, then the connection induced by $\mu$ is of the first kind.

Proof. This uses the result from [8] that if $g=m+h$ as above and $[m m]_{m} \neq 0$, then the algebra $(m, 1 / 2 X Y)$ is simple.

\section{REFERENCES}

1. S. Helgason, Differential Geometry and Symmetric Spaces, Academic Press, New York, 1962.

2. E. Hille and R. Phillips, Functional Analysis and Semi-Groups, Amer. Math. Soc. Providence, R. I., 1957.

3. N. Jacobson, Lie Algebras, Interscience, New York, 1962.

4. S. Kobayashi and K. Nomizu, Foundations of Differential Geometry I and II, Interscience, New York, 1963.

5. S. Lang, Analysis I, Addison-Wesley, Reading, Mass., 1968.

6. K. Nomizu, Invariant affine connections on homogeneous spaces, Amer. J. Math., 76 (1954), 33-65.

7. - Recent development in the theory of connections and holonomy groups, Advances in Mathematics, Academic Press, New York, 1961.

8. A. Sagle and D. Winter, On homogeneous spaces and reductive subalgebras of simple Lie algebras, Trans. Amer. Math. Soc., 128 (1967), 142-147. 
9. J. Tits, Liesche Gruppen und Algebren, Der Universitat Bonn, Bonn, 1965.

Received February 17, 1971 and in revised form June 6, 1972. This research was supported in part by NSF Grants GP-13328 and GP-29224.

UNIVERSITY OF MINNESOTA

AND

University of Hawait, Hilo College 


\section{PACIFIC JOURNAL OF MATHEMATICS}

\section{EDITORS}

RICHARD ARENS (Managing Editor)

University of California

Los Angeles, California 90024

R. A. Beaumont

University of Washington

Seattle, Washington 98105
J. DUGUNDJI*

Department of Mathematics

University of Southern California

Los Angeles, California 90007

D. Gilbarg and J. Milgram

Stanford University

Stanford, California 94305

\section{ASSOCIATE EDITORS}
E. F. BECKENBACH
B. H. NeumanN
F. WOLF
K. YosHIDA

\section{SUPPORTING INSTITUTIONS}

UNIVERSITY OF BRITISH COLUMBIA
CALIFORNIA INSTITUTE OF TECHNOLOGY
UNIVERSITY OF CALIFORNIA
MONTANA STATE UNIVERSITY
UNIVERSITY OF NEVADA
NEW MEXICO STATE UNIVERSITY
OREGON STATE UNIVERSITY
UNIVERSITY OF OREGON
OSAKA UNIVERSITY

UNIVERSITY OF BRITISH COLUMBIA

UNIVERSITY OF CALIFORNIA

MONTANA STATE UNIVERSITY

UNIVERSITY OF NEVADA

OREGON STATE UNIVERSITY

OSAKA UNIVERSITY
UNIVERSITY OF SOUTHERN CALIFORNIA

STANFORD UNIVERSITY

UNIVERSITY OF TOKYO

UNIVERSITY OF UTAH

WASHINGTON STATE UNIVERSITY

UNIVERSITY OF WASHINGTON

\section{AMERICAN MATHEMATICAL SOCIETY} NAVAL WEAPONS CENTER

* C. R. DePrima California Institute of Technology, Pasadena, CA 91109, will replace J. Dugundji until August 1974. 


\section{Pacific Journal of Mathematics}

\section{Vol. 48, No. $1 \quad$ March, 1973}

Jan Aarts and David John Lutzer, Pseudo-completeness and the product of Baire

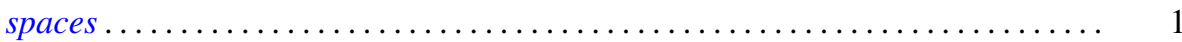

Gordon Owen Berg, Metric characterizations of Euclidean spaces ............ 11

Ajit Kaur Chilana, The space of bounded sequences with the mixed topology ..... . 29

Philip Throop Church and James Timourian, Differentiable open maps of

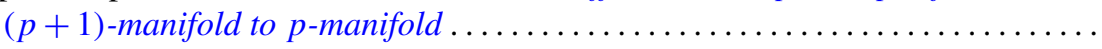

P. D. T. A. Elliott, On additive functions whose limiting distributions possess a finite

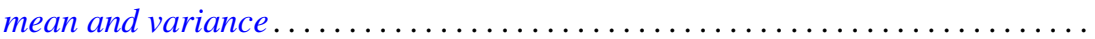

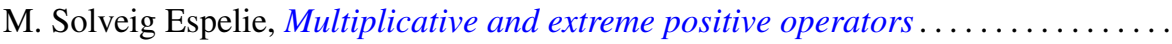

Jacques A. Ferland, Domains of negativity and application to generalized convexity

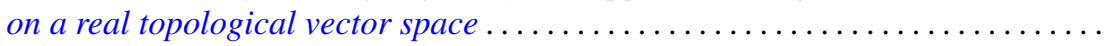

Michael Benton Freeman and Reese Harvey, A compact set that is locally holomorphically convex but not holomorphically convex ...............

Roe William Goodman, Positive-definite distributions and intertwining

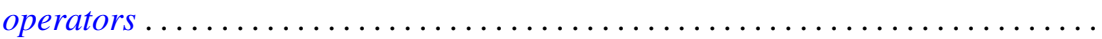

Elliot Charles Gootman, The type of some $C^{*}$ and $W^{*}$-algebras associated with

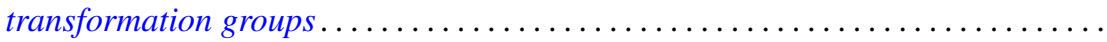

David Charles Haddad, Angular limits of locally finitely valent holomorphic

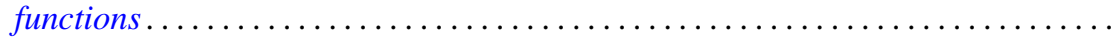

William Buhmann Johnson, On quasi-complements .

William M. Kantor, On 2-transitive collineation groups of finite projective spaces...

Joachim Lambek and Gerhard O. Michler, Completions and classical localizations of right Noetherian rings

Kenneth Lamar Lange, Borel sets of probability measures ......

David Lowell Lovelady, Product integrals for an ordinary differential equation in a Banach space

Jorge Martinez, A hom-functor for lattice-ordered groups .........

W. K. Mason, Weakly almost periodic homeomorphisms of the two sphere ....

Anthony G. Mucci, Limits for martingale-like sequences .......

Eugene Michael Norris, Relationally induced semigroups ...

Arthur E. Olson, A comparison of c-density and $k$-density ......

Donald Steven Passman, On the semisimplicity of group rings of linear groups.

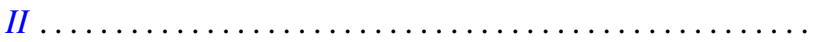

Charles Radin, Ergodicity in von Neumann algebras .

P. Rosenthal, On the singularities of the function generated by the Bergman operator of the second kind.

Arthur Argyle Sagle and J. R. Schumi, Multiplications on homogeneous spaces,

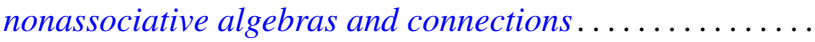

Leo Sario and Cecilia Wang, Existence of Dirichlet finite biharmonic functions on

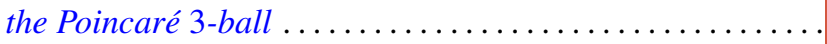

Ramachandran Subramanian, On a generalization of martingales due to Blake ..

Bui An Ton, On strongly nonlinear elliptic variational inequalities.

Seth Warner, A topological characterization of complete, discretely valued

fields. 